\title{
Smartphone usage during meals is a potential risk for weight gain in post-adolescent female students
}

\author{
Tomoko Fujiwara ${ }^{1 *}$ and Rieko Nakata ${ }^{2}$ \\ ${ }^{1}$ Department of Home Science and Welfare, Kyoto Notre Dame University, Kyoto, Japan \\ ${ }^{2}$ Department of Food Science and Nutrition, Nara Women's University, Nara, Japan
}

\begin{abstract}
Recent studies demonstrated that intervention using a mobile phone was associated with significant changes in the body weight and body mass index by providing a self-monitoring program of dietary intake.However, there are very few reports concerning the direct influence of smartphone use on food intake or the reproductive function. Therefore, we examined this issue by conducting a questionnaire survey of 213 Japanese female college students aged from $18-20$ years old. There was no significant relationship between smartphone use during meals and menstrual disorders.In contrast, the incidence of body weight gain was significantly higher in populations who use a smartphone during meals than students who did not use a smartphone while eating.Although the precise mechanism remains unclear, this study newly provides important and cautionary evidence that smartphone use during meals may interfere with an adequate control of food intake by adversely affecting appetite.
\end{abstract}

\section{Introduction}

The dietary habit is one of the key factors that influence human lifestyles and individuals' quality of life (QOL). It is well-known that representative menstrual disorders such as irregular menstruation and dysmenorrhea can be caused by inappropriate dietary habits [1]. The consumption of fast food and the habit of skipping meals is wide spread among young Japanese women [2]. Previously, we reported that young women who skip breakfast have a significantly higher incidence of dysmenorrhea than those who eat breakfast [3], suggesting a positive correlation between skipping breakfast and menstrual disorders. It was also demonstrated that young females consuming fast and processed foods have a high incidence of dysmenorrhea [4]. On the other hand, we observed that the frequency of irregular menstruation is increased in young women who are currently on a diet, while the intensity of dysmenorrhea is high in those with a history of dieting in adolescence, providing cautionary evidence that dieting in adolescence has longlasting adverse effects on the reproductive function in young women [5]. From these findings, we deduced that dietary habits during both adolescent and post-adolescent stages are important factors that subsequently influence the reproductive function in the future [6].

Recent studies demonstrated that mobile phone intervention was associated with significant changes in the body weight and body mass index [7]. It was also demonstrated that self-monitoring of dietary intake using smartphone is as accurate as paper-based records but more acceptable to young women [8]. Smartphone applications for weight loss programs were reported to effectively support weight loss in women who were overweight and obese [9]. Consequently, mobile technologies were proposed to promote healthy weight-related behaviors in young adults [10].

Mobile phones have widely spread among young students in Japan. Although the usage of smartphones for self-monitoring of the dietary intake or a program for weight loss is now under investigation in the world, the direct influence of smartphone use on food intake or the reproductive function has not yet been evaluated. Based on this background, we hypothesized that using a smartphone during meals has an adverse influence on physical conditions, and examined the relationship between smartphone use during a meal and menstrual disorders or body weight gain in post-adolescent female students by conducting a questionnaire survey.

\section{Methods}

\section{Respondents to a questionnaire}

The subjects were young Japanese women aged from 18-20 years old who studied at the Faculty of Home Economics of Ashiya College, Kyoto Bunkyo Junior College, and Nara Women's University. The study protocol was approved by the Committee on Food Culture at Ashiya College. We sent questionnaires to 234 students. Information regarding the aim of this study was sent with the questionnaire, and consent was obtained from all participants.

\section{Questionnaire items}

1) Use of smartphones during meals: The participants were divided into two groups as follows: Group I: using a smartphone during meals; and Group II: not using a smartphone during meals.

2) Dysmenorrhea: Dysmenorrhea was graded as previously described by Fujiwara [3]. Grade 1 (free of pain or painful, but can

Correspondence to: Tomoko Fujiwara, Department of Home Science and Welfare, Kyoto Notre Dame University, Kyoto, Japan, Tel: 81757063688; Fax: 81757063707; E-mail: fujiwara@notredame.ac.jp; tomokof@bd5.so-net.ne.jp

Key words: appetite, dysmenorrhea, irregular menses, smartphone, weight gain, young women

Received: October 04, 2016; Accepted: October 31, 2016; Published: November 02,2016 
manage without an analgesic), Grade 2 (painful, requiring an analgesic), and Grade 3 (painful, not relieved by an analgesic).

3) Irregular menstruation: In addition, the participants were divided into 2 groups depending on whether they had regular or irregular menstruation. Regular menstruation was strictly defined as constant 26-32-day intervals in each menstrual cycle, as previously described by Fujiwara [3].

4) Body mass index (BMI) assessment: Information on the body mass (kilograms) and height (meters) was obtained from all participants. The BMI was calculated using the formula: body weight in kilograms divided by height in meters squared.

5) Gain of body weight: The participants were divided into 2 groups depending on whether or not they had experienced $3-\mathrm{kg}$ increase in their body weight during the last 3 years.

\section{Statistical analysis}

The data are shown as the mean \pm standard deviation (SD). Differences in the grades of dysmenorrhea and the BMI values between Groups I and II were analyzed by the Mann-Whitney test. Differences in the incidence of irregular menstruation and body weight gain between Groups I and II were analyzed by the Chi square test.P-values less than 0.05 were significant.

\section{Results}

From the 234 students to whom we sent questionnaires, we obtained 213 responses that were suitable for statistical analysis. Among these 213 students, 143 (67.1\%) used a smartphone during meals (Group I), whereas the other 70 students (32.9\%) did not use a smartphone while eating (Group II).

There was no significant difference in the grades of dysmenorrhea between Groups I and II (Table 1). Similarly, there was no significant difference in the incidence of irregular menses between the two groups (Table 1).

On the other hand, although there were no significant differences in the BMI between Groups I and II $(19.96 \pm 2.23$ and $19.94 \pm 2.28$, respectively), the incidence of body weight gain in Group I was significantly higher (59.4\%) than that in Group II (37.1\%) (Table 1).

\section{Discussion}

The present study demonstrated that about two thirds of young female students in post-adolescence had a habit of using a smartphone

Table 1. The relationship between smartphone usage during meals and dysmenorrhea, the incidence of menstrual cycle irregularity or weight gain.

\begin{tabular}{|c|c|c|c|}
\hline & Group I & Group II & \\
\hline \multicolumn{4}{|c|}{ Dysmenorrhea } \\
\hline Scores & $1.14 \pm 0.52$ & $1.11 \pm 0.58$ & (Not significant) \\
\hline \multicolumn{4}{|c|}{ Menstrual cycle } \\
\hline Regular & 67 & 32 & \\
\hline Irregular & 76 & 38 & (Not significant) \\
\hline \multicolumn{4}{|c|}{ Weight gain } \\
\hline Positive & 85 & 26 & \\
\hline Negative & 58 & 44 & $(\mathrm{P}<0.01)$ \\
\hline
\end{tabular}

Group I: using a smartphone during meals; Group II: not using a smartphone during meals. There was no significant difference in the grades of dysmenorrhea or the incidence of irregular menses between Groups I and II. On the other hand, although there were no significant differences in the BMI between Groups I and II, the incidence of body weight gain in Group I was significantly higher than that in Group II. during meals in Japan. There was no significant relationship between smartphone use during meals and representative menstrual disorders such as irregular menstruation and dysmenorrhea. In contrast, the incidence of body weight gain was significantly higher in those who use a smartphone during meals. As far as we know, this is the first report to focus on smartphone use during meals and examine its relationship with changes in the body weight.

In Japan, adolescents tend to try to lose body weight for cosmetic reasons, and have become conscious of how to control their body weight.Indeed, we previously confirmed that more than $60 \%$ of Japanese college students used diet control to reduce their body weight and about $40 \%$ of students had undergone dietary restriction in adolescence [5]. However, in Japan, daily lifestyles including dietary habits change after entering university or college and marked adaptations are needed in post-adolescent students. This change may lead to poor self-control of their body weight. In accordance with this speculation, this study revealed that 111 of 213 participants (52.1\%) had experienced more than $3-\mathrm{kg}$ body weight gain over the last 3 years.

The precise reasons why those using a smartphone during meals have a higher incidence of body weight gain than the non-using group remain unclear. Taste affects food intake along with other sensory cues such as appearance, smell, texture, temperature, and flavor [11].In the brain, the system of taste cooperates with the visual, olfactory, somatosensory, and auditory sensory systems [12] and plays an important role in the control of appetite [13]. Among them, the first sensory contact with food is through the eyes in most cases. Accordingly, using a smartphone during a meal is considered to adversely affect taste by reducing visual effects of the food on taste.In addition, it may interfere with other sensory inputs when enthusiastic about handling a smartphone. These aspects can cause a significant delay of pleasantness, resulting in excess food intake.

\section{Conclusions}

In conclusion, this study demonstrated that smartphone use during meals is associated with body weight gain. Although the precise mechanism remains unknown, this study newly provides important and cautionary evidence that smartphone use during meals may adversely affect the appetite, interfering with adequate control of food intake.

\section{Acknowledgements}

The authors do appreciate Prof. Hiroshi Fujiwara, MD for his advice of medical discussion and Ms.Fumino Yamagishi for her support of statistical analysis.

\section{Disclosure of interest}

The authors report no conflicts of interest.This work was supported in part by a Grant-in-Aid for Scientific Research (No. 15K00903).

\section{References}

1. Nazni P (2014) Association of western diet and lifestyle with decreased fertility. Indian $J$ Med Res 140: S78-S81. [Crossref]

2. Murata M (1992) Nutrition for the young-its current problems. Nutr Health 8: 143-152. [Crossref]

3. Fujiwara T (2003) Skipping breakfast is associated with dysmenorrhea in young women in Japan. Int J Food Sci Nutr 54: 505-509. [Crossref]

4. Fujiwara T, Sato N, Awaji H, Sakamoto H, Nakata R (2009) Skipping breakfast adversely affects menstrual disorders in young college students. Int J Food Sci Nutr 60: 23-31. [Crossref] 
Fujiwara T (2016) Smartphone usage during meals is a potential risk for weight gain in post-adolescent female students

5. Fujiwara $\mathrm{T}$ (2007) Diet during adolescence is a trigger for subsequent development of dysmenorrhea in young women. Int J Food Sci Nutr 58: 437-444. [Crossref]

6. Fujiwara T, Nakata R (2010) Skipping breakfast is associated with reproductive dysfunction in post-adolescent female college students. Appetite 55: 714-717. [Crossref]

7. Liu F, Kong X, Cao J, Chen S, Li C, et al. (2015) Mobile phone intervention and weight loss among overweight and obese adults: a meta-analysis of randomized controlled trials. Am J Epidemiol 181: 337-348. [Crossref]

8. Hutchesson MJ, Rollo ME, Callister R, Collins CE (2015) Self-monitoring of dietary intake by young women: online food records completed on computer or smartphone are as accurate as paper-based food records but more acceptable. J Acad Nutr Diet 115 : 87-94. [Crossref]
9. Pellegrini CA, Pfammatter AF, Conroy DE, Spring B (2015) Smartphone applications to support weight loss: current perspectives. Adv Health Care Technol 1: 13-22. [Crossref]

10. Patrick K, Marshall SJ, Davila EP, Kolodziejczyk JK, Fowler JH, et al. (2014) Design and implementation of a randomized controlled social and mobile weight loss trial for young adults (project SMART). Contemp Clin Trials 37: 10-18. [Crossref]

11. Wadhera D, Capaldi-Phillips ED (2014) A review of visual cues associated with food on food acceptance and consumption. Eat Behav 15: 132-143. [Crossref]

12. Doty RL (2012) Gustation. Wiley Interdiscip Rev Cogn Sci 3: 29-46. [Crossref]

13. Rolls ET (2015) Taste, olfactory, and food reward value processing in the brain. Prog Neurobiol 128: 64-90. [Crossref]

Copyright: (C2016 Fujiwara T. This is an open-access article distributed under the terms of the Creative Commons Attribution License, which permits unrestricted use, distribution, and reproduction in any medium, provided the original author and source are credited. 